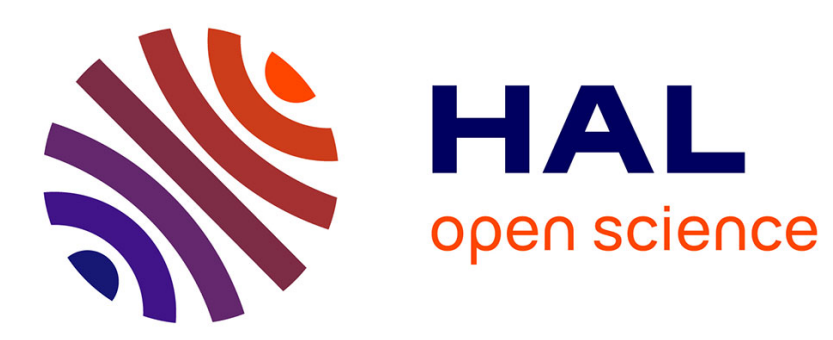

\title{
Une nouvelle méthode de mesure des temps de relaxation très courts en résonance électronique : la méthode goniométrique
}

\author{
Henri Place
}

\section{- To cite this version:}

Henri Place. Une nouvelle méthode de mesure des temps de relaxation très courts en résonance

électronique: la méthode goniométrique. Revue de Physique Appliquée, 1968, 3 (2), pp.203-208. 10.1051/rphysap:0196800302020300 . jpa-00242846

\section{HAL Id: jpa-00242846 \\ https://hal.science/jpa-00242846}

Submitted on 1 Jan 1968

HAL is a multi-disciplinary open access archive for the deposit and dissemination of scientific research documents, whether they are published or not. The documents may come from teaching and research institutions in France or abroad, or from public or private research centers.
L'archive ouverte pluridisciplinaire HAL, est destinée au dépôt et à la diffusion de documents scientifiques de niveau recherche, publiés ou non, émanant des établissements d'enseignement et de recherche français ou étrangers, des laboratoires publics ou privés. 


\title{
UNE NOUVELLE MÉTHODE DE MESURE DES TEMPS DE RELAXATION TRÈS GOURTS EN RÉSONANGE ÉLEGTRONIQUE : LA MÉTHODE GONIOMÉTRIQUE (1)
}

\author{
Par HenRi PLACE, \\ Institut d'Électronique Fondamentale, Laboratoire associé au C.N.R.S., Faculté des Sciences, Orsay.
}

(Reçu le 20 février 1968.)

\begin{abstract}
Résumé. - Dans cette méthode, le champ d'hyperfréquence est modulé en amplitude. Les variations résultantes de l'aimantation longitudinale à la fréquence de modulation sont représentées par un vecteur de Fresnel, dont l'extrémité décrit une courbe dans le plan complexe lorsque l'on fait varier le champ directeur en passant par la valeur de résonance. On montre que les valeurs de $T_{1}$ et de $T_{2}^{*}$ sont déduites de mesures angulaires simples faites sur cette courbe. La mise en ouvre expérimentale de cette méthode est décrite et ses caractéristiques principales sont données : précision (5 à $10 \%$ ), gamme des temps mesurés $\left(10^{-5}\right.$ à $\left.5 \times 10^{-10} \mathrm{~s}\right)$ et sensibilité.
\end{abstract}

Abstract. - We describe a new method of electronic relaxation time measurement, using an amplitude modulated U.H.F. field. The resulting variations of longitudinal magnetization at the modulation frequency are represented by a Fresnel vector. Its extremity describes a curve in the complex plane, when the external magnetic field is swept through the resonance value. The $T_{1}$ and $T_{2}^{*}$ values are easily deduced from angular measurements on this curve. The experimental set-up is described and its main characteristics are discussed : accuracy $\left(5-10 \%\right.$ ), range of measured relaxation times (from $10^{-5}$ to $5 \times 10^{-10} \mathrm{~s}$ ) and sensitivity.

Il existe de nombreuses méthodes de mesure des temps de relaxation en résonance électronique, mais la plupart sont limitées à la mesure de temps supérieurs à la microseconde. Seules la mesure du temps de relaxation spin-spin $T_{2}$ (ou $T_{2}^{*}$ pour les raies inhomogènes) par mesure de la largeur de raie ou la mesure du temps de relaxation spin-réseau $T_{1}$ par saturation progressive de la résonance ne connaissent théoriquement pas de limite vers les temps très courts, mais leur précision est médiocre. La méthode que nous proposons est fondée sur le même principe que celle de Hervé et Pescia [1], [2], qui a permis de mesurer des temps $T_{1}$ compris entre $10^{-6}$ et $10^{-8} \mathrm{~s}$; mais en améliorant sa précision, sa rapidité et l'étendue de sa gamme de mesure par un traitement plus général, nous l'avons transformée en une méthode entièrement nouvelle.

1. Exposé de la méthode. - Le principe de la méthode goniométrique est le suivant : on excite la résonance électronique de l'échantillon par un champ d'hyperfréquence modulé en amplitude. Les variations résultantes de l'aimantation longitudinale $M_{\mathrm{z}}$

(1) Cet article recouvre en partie la thèse de Doctorat ès Sciences Physiques présentée par l'auteur à la Faculté des Sciences d'Orsay le 11 décembre 1967 devant la Commission d'examen : M. Grivet, président; MM. Froidevaux, Vautier, Hervé, examinateurs. Numéro d'enregistrement C.N.R.S. : A.O. 1894. à la fréquence de modulation sont recueillies àm l'aide d'une petite bobine et constituent le signal. On étudie alors la « courbe de résonance » décrite, dans le plan complexe, par l'extrémité du vecteur de Fresnel représentant ce signal, lorsque le champ directeur varie en passant par la valeur de résonance. C'est à partir de mesures angulaires très simples faites sur cette courbe que l'on détermine les temps de relaxation $T_{1}$ et $T_{2}^{*}$.

1.1. Mise en ÉQuATIONS. - L'équation utilisée ici est l'équation de Bloch, qui décrit la relaxation par deux temps $T_{1}$ et $T_{2}$. Elle s'écrit :

$\frac{\mathrm{d} \mathbf{M}}{\mathrm{d} t}=\gamma \mathbf{M} \wedge\left(\mathbf{H}_{\mathbf{0}}+\mathbf{H}_{1}\right)-\frac{M_{\mathrm{z}}-M_{\mathbf{0}}}{T_{\mathbf{1}}}-\frac{M_{\perp}}{T_{\mathbf{2}}}$.

Les notations ont la signification suivante :

$M$ : aimantation instantanée,

$M_{0}$ : aimantation à l'équilibre,

$M_{\mathrm{z}}$ : composante de $M$ parallèle au champ directeur,

$M_{\perp}$ : composante de $M$ perpendiculaire au champ directeur,

$H_{0}$ : champ directeur orienté suivant $\mathrm{Oz}$,

$H_{1}$ : champ d'hyperfréquence perpendiculaire à $H_{0}$, modulé en amplitude à la fréquence $F=\Omega / 2 \pi$ avec un taux de modulation $k$.

En projection sur les axes du trièdre tournant à la vitesse angulaire $\omega$ (pulsation du champ d'hyperfréquence $H_{1}$ ), cette équation donne les valeurs station- 
naires habituelles de l'absorption, de la dispersion et de l'aimantation longitudinale $M_{\mathrm{z}}$.

En écrivant que la modulation d'amplitude agit comme une perturbation sur ces solutions stationnaires et en limitant le calcul au premier ordre $(k \ll 1)$, le signal cherché, dû à cette perturbation, est donné par [1] :

$$
\begin{aligned}
s= & -\frac{j a k M_{0}}{1+a+\delta^{2}} \\
& {\left[\frac{(1+j Y)^{2}+\delta^{2}+\left(1+\delta^{2}\right)(1+j Y)}{a(1+j Y)+(1+j X)\left[(1+j Y)^{2}+\delta^{2}\right]}\right] . }
\end{aligned}
$$

En supposant $a \ll 1$, ce qui est toujours le cas en pratique, l'expression (2) s'écrit finalement :

$s=-a k M_{0} \Omega\left(\frac{1}{X-j}\right)\left(\frac{1}{1+\delta^{2}}+\frac{1+j Y}{(1+j Y)^{2}+\delta^{2}}\right)$

ou encore :

$$
s=C \cdot \frac{1}{X-j} \cdot f\left(Y, \delta^{2}\right) .
$$

La méthode goniométrique est fondée sur l'enregistrement de la " courbe de résonance » décrite par l'image de $s$ dans le plan complexe, lorsque $\delta$ varie. Nous allons étudier l'allure de cette courbe que nous appellerons $\mathrm{S}$, suivant les valeurs de $X$ et de $Y$.

1.2. ÉTUDE DES « GOURBES DE RÉSONANGE ». - Il faut tout d'abord remarquer que, d'après l'expression (4), la forme des courbes $\mathrm{S}$ est donnée par la fonction $f$ (qui seule dépend de $\delta$ ). Cette forme ne dépend donc que de $Y$, c'est-à-dire de $T_{2}$. Le terme $1 /(X-j)$, qui est un facteur de similitude, n'intervient que pour fixer la position angulaire, par rapport aux axes du plan complexe, des courbes données par la fonction $f$, que nous appellerons courbes $\mathrm{F}$. Les courbes $\mathrm{F}$ et les courbes $\mathrm{S}$ sont donc similaires dans leur forme (à une homothétie près), mais ont des positions angulaires différentes qui ne dépendent que de $X$, donc de $T_{1}$.

Nous étudions d'abord l'évolution de la forme des courbes $\mathrm{F}$ en fonction de $Y$. La fonction :

$$
f=\frac{1}{1+\delta^{2}}+\frac{1+j Y}{(1+j Y)^{2}+\delta^{2}}
$$

est la somme de deux termes qui représentent le premier une droite et le second un cercle. Lorsque $Y$ varie de 0 à l'infini, les courbes $\mathrm{F}$ passent de la forme d'une droite (pour $Y$ petit, $f$ est équivalent à $2 /\left(1+\delta^{2}\right)$ ) à celle d'un cercle et d'une droite (qui est le diamètre du cercle). Cette dernière forme est obtenue pour $Y$ très grand : en effet, lorsque $\delta$ est également très grand, $f$ se réduit au second terme qui représente le cercle et, lorsque $\delta$ est petit, le premier terme est prépondérant et donne l'axe réel limité à +1 (double du diamètre du cercle). Il vient donc à l'idée que de telles courbes peuvent être des cubiques circulaires. En ramenant l'origine des coordonnées au point double $\left(\frac{1}{2},-\frac{1}{Y}\right)$ de la courbe $\mathrm{F}$, on obtient l'équation polaire :

$\rho=\frac{2}{Y\left(Y^{2}+4\right) \sin \theta}-\frac{\sqrt{Y^{2}+1}}{2 Y} \cos (\theta-\alpha)$

avec :

$$
\operatorname{tg} \alpha=-\frac{3 Y^{2}+4}{Y^{3}} .
$$

G'est bien l'équation d'une cubique circulaire. On en déduit la construction suivante : chaque point d'une courbe $\mathrm{F}$ est obtenu comme différence des distances entre un point $d$ de coordonnées $\left(\frac{1}{2},-\frac{1}{Y}\right)$ situé sur un cercle de rayon $\frac{\sqrt{Y^{2}+1}}{4 Y}$ et de centre $\left(\frac{3 Y^{2}+8}{4\left(Y^{2}+4\right)},-\frac{7 Y^{2}+20}{4 Y\left(Y^{2}+4\right)}\right)$, et d'une $\underset{Y^{2}+2}{\text { part une }}$ droite $\Delta$ parallèle à l'axe réel d'ordonnée $-\frac{Y^{2}+2}{Y\left(Y^{2}+4\right)}$, d'autre part le cercle précédent, cette différence étant prise à partir du point $d$. Les courbes $\mathrm{F}$ sont limitées d'un côté à l'origine des coordonnées et de l'autre côté au point de coordonnées :

$$
\left(\frac{Y^{2}+2}{Y^{2}+1},-\frac{Y}{Y^{2}+1}\right) \text {. }
$$

Les figures 1 et 2 montrent deux exemples de formes que peuvent prendre les courbes $\mathrm{F}$ suivant les valeurs du paramètre $Y$. C'est à partir de considérations géométriques simples faites sur ces courbes que nous avons déterminé les temps de relaxation $T_{1}$ et $T_{2}$.

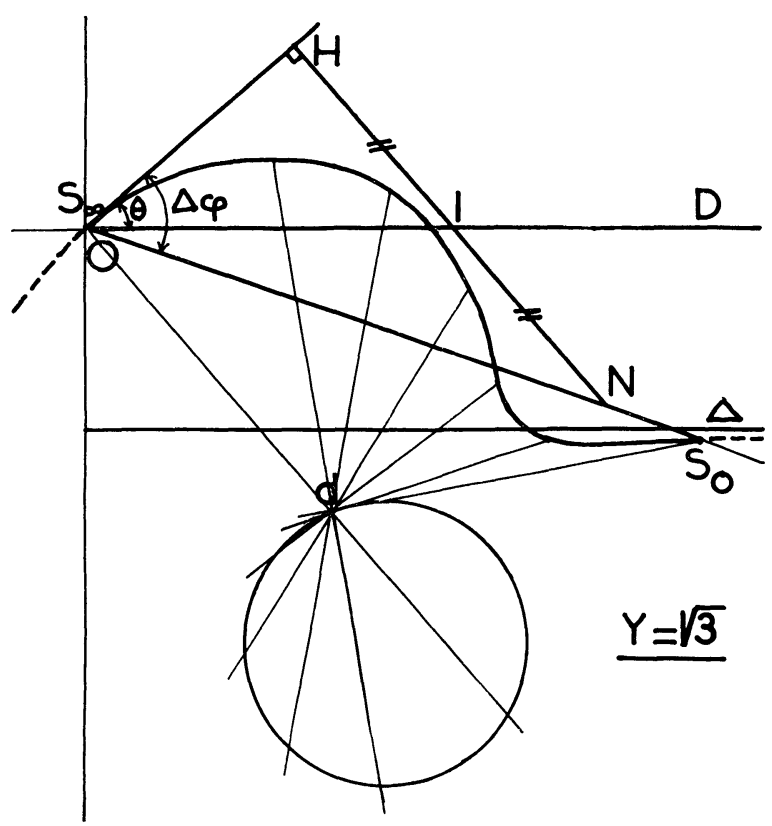

FIG. 1. - Construction de la courbe F et de la droite D. 


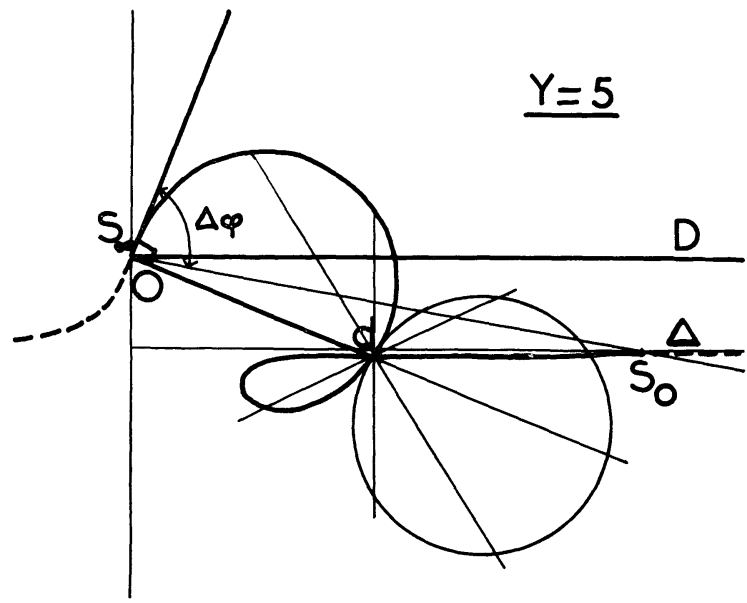

FIG. 2.

Construction de la courbe $\mathrm{F}$ et de la tangente à l'origine.

1.3. DÉtermination de $T_{2}$ (GAS homogène). - Les courbes $\mathrm{S}$ (ou $\mathrm{F}$ ) sont obtenues expérimentalement en faisant varier lentement le champ directeur, donc $\delta$. $f$ étant une fonction paire de $\delta$, un même point est obtenu pour deux valeurs opposées de $\delta$. Les courbes $\mathrm{S}$ sont donc limitées d'un côté à l'origine des axes $S_{\infty}$ correspondant à $\delta= \pm \infty$ ou à $H_{0}=0$ ou $+\infty$ et de l'autre côté au point $S_{0}$ correspondant à $\delta=0$ (résonance). Appelons $\varphi_{\infty}$ et $\varphi_{0}$ les arguments respectifs de la tangente à l'origine et du rayon vecteur $\mathbf{S}_{\infty} \mathbf{S}_{\mathbf{0}}$. On peut écrire :

$$
\begin{aligned}
\operatorname{tg}\left(\varphi_{\infty}-\varphi_{0}\right) & =\operatorname{tg} \Delta \varphi=\operatorname{tg}\left(\operatorname{Arg} \frac{f_{\infty}}{f_{0}}\right) \\
& =\operatorname{tg}\left(\operatorname{Arg} \frac{2+j Y}{\delta^{2}} \cdot \frac{1+j Y}{2+j Y}\right)=Y .
\end{aligned}
$$

Ainsi la mesure de $\Delta \varphi$ (angle de la tangente à l'origine de la courbe $\mathrm{S}$ avec le rayon vecteur de son extrémité), à une fréquence de modulation donnée, permet de déterminer $T_{2}$ à partir de la relation (7), soit :

$$
T_{2}=\operatorname{tg} \Delta \varphi / \Omega
$$

Lorsque la « courbe de résonance » présente un point double, on peut construire la tangente à l'origine en menant la perpendiculaire au rayon vecteur du point double (fig. 2).

1.4. Détermination de $T_{1}$. Considérons maintenant la droite $\mathrm{D}$ joignant l'origine au point d'affixe $1 /(X-j)$. Cette droite fait avec l'axe imaginaire l'angle $\varphi$ tel que :

$$
\operatorname{tg} \varphi=X
$$

On voit que si l'on peut déterminer cette droite $\mathrm{D}$ à partir de constructions simples faites sur la courbe $\mathrm{S}$, la valeur de $X$ s'en déduira aussitôt et donc la valeur de $T_{1}$. Calculons alors l'angle $\theta$ que fait cette droite $\mathrm{D}$ avec la tangente à l'origine. On a :

$$
\begin{aligned}
\theta=\operatorname{Arg} s_{\infty}- & \operatorname{Arg} \frac{1}{X-j} \\
& =\operatorname{Arg}\left[s_{\infty}(X-j)\right]=\operatorname{Arg}\left(C \frac{2+j Y}{\delta^{2}}\right)
\end{aligned}
$$

d'où :

$$
\operatorname{tg} \theta=\frac{Y}{2}=\frac{1}{2} \operatorname{tg} \Delta \varphi
$$

Cette formule montre que la droite D est liée intrinsèquement à la courbe $\mathrm{S}$. On peut la construire en joignant l'origine au milieu de la perpendiculaire abaissée d'un point quelconque du rayon vecteur $\mathbf{S}_{\infty} \mathbf{S}_{0}$ sur la tangente à l'origine ( fig. 1).

Une autre construction plus simple est également possible : on montre facilement que la droite $D$ passe par le point où la médiatrice du rayon vecteur $\mathbf{S}_{\infty} \mathbf{S}_{\mathbf{0}}$ coupe la courbe $\mathrm{S}$ ( fig. 1). Enfin, la direction de la droite $\mathrm{D}$ est donnée également par l'asymptote des courbes $\mathrm{S}$ lorsque $\delta \rightarrow 0$ ( fig. 1 et 2 ).

La droite $\mathrm{D}$ étant construite par l'un quelconque de ces procédés, il suffit de mesurer l'angle $\varphi$ pour obtenir $T_{1}$ par la formule $(9)$, c'est-à-dire :

$$
T_{1}=\operatorname{tg} \varphi / \Omega \text {. }
$$

1.5. Cas D'Un ÉLARgissement inhomogène DE LA RAIE. - Tous les calculs précédents ont été faits en supposant que la largeur de la raie était de nature homogène. S'il en est autrement, il faut revoir les calculs pour tenir compte de l'élargissement inhomogène de la raie. On suppose alors que la raie est constituée par la superposition de raies homogènes, avec une distribution définie par une certaine fonction $g\left(\omega_{0}\right)$. Le signal s'écrit alors sous la forme du produit de convolution :

$$
s^{*}=s * g \text {. }
$$

Le calcul de $s^{*}$ ne conduit à des résultats simples et facilement exploitables que dans deux cas : celui où la fonction $g\left(\omega_{0}\right)$ est lorentzienne (puisque $s$ apparaît en (3) comme la somme de deux fonctions de Lorentz) et celui où la largeur de la fonction de distribution est bien supérieure à celle de la raie homogène.

Dans le premier cas, le produit de convolution de deux fonctions lorentziennes étant encore une fonction lorentzienne, on trouve que $s^{*}$ se met sous la forme :

$s^{*}=C^{*} \frac{1}{X-j}\left(\frac{1}{1+\delta^{* 2}}+\frac{1+j Y^{*}}{\left(1+j Y^{*}\right)^{2}+\delta^{* 2}}\right)$

où $\quad C^{*}=\frac{T_{2}^{*}}{T_{2}} G, \quad \delta^{*}=\left(\omega-\omega_{0}\right) T_{2}^{*}$ et $Y^{*}=\Omega T_{2}^{*}$ ( $T_{2}^{*}$ définit la largeur de la raie inhomogène). On remarque que l'expression (12) est formellement identique à (3), à condition de remplacer $T_{2}$ par $T_{2}^{*}$; la détermination de $T_{1}$ n'est donc pas modifiée et la méthode goniométrique permet de mesurer $T_{1}$ et $T_{2}^{*}$. 
Dans le second cas, le signal s'écrit sensiblement :

$$
s^{*}=g\left(\omega_{0}\right) \int_{-\infty}^{+\infty} s\left(\delta^{2}\right) \mathrm{d} \delta=\frac{2 \pi C}{X-j} g\left(\omega_{0}\right) .
$$

La courbe $\mathrm{S}$ est donc une droite confondue avec la droite $\mathrm{D}$, ce qui permet de déterminer simplement $T_{1}$. Ce cas se rencontre couramment dans l'étude de poudres ferromagnétiques.

2. Míse en œuvre de la méthode. - Les expériences ont été faites dans trois bandes d'hyperfréquences différentes $(S, X$ et $K a)$. Aussi nous décrirons d'abord brièvement la partie U.H.F. de l'appareillage, qui constitue la production du signal $s$ et qui diffère suivant la bande d'hyperfréquences utilisée. La partie H.F. et B.F. de l'appareillage qui sert à l'amplification du signal et à son enregistrement sera analysée ensuite : elle est commune aux trois bandes d'hyperfréquences. Le schéma de principe de l'appareillage est représenté sur la figure 3 .

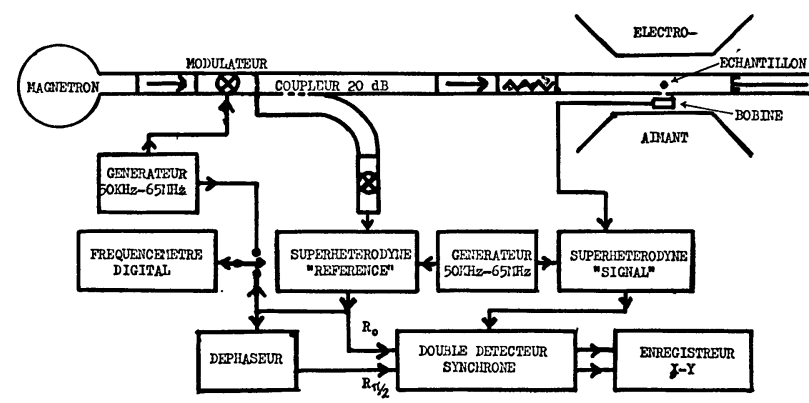

Frg. 3. - Schéma de principe de l'appareillage nécessaire à la méthode goniométrique.

2.1. Production du signal. - Quelle que soit la bande d'hyperfréquences utilisée, on retrouve dans les trois montages des éléments essentiels différents dont la fonction est identique. Il s'agit de la source d'hyperfréquences, du modulateur d'amplitude, de la référence de phase et de l'extraction du signal.

2.1.1. Source d'hyperfréquences. - La source doit être d'une puissance suffisante (de l'ordre de quelques watts) pour éviter l'emploi, comme organe d'excitation de la résonance de l'échantillon, d'une cavité résonnante : en effet, aux fréquences de modulation très élevées (jusqu'à $100 \mathrm{MHz}$ ) nécessaires pour mesurer des temps de relaxation très courts, la bande passante étroite de la cavité risque d'introduire un déphasage des bandes latérales et d'entacher les mesures d'une grave erreur.

Nous avons donc utilisé en bande $S$ (de 2 à $4,4 \mathrm{GHz}$ ) un générateur U.H.F. suivi d'un tube à onde progressive, en bande $X$ (de 8,7 à $9,7 \mathrm{GHz}$ ) un magnétron accordable, et en bande $K a$ (de 35 à $37 \mathrm{GHz}$ ) un klystron accordable.

2.1.2. Modulateur d'amplitude. - Nous avons adopté comme principe du modulateur d'amplitude le seul qui permette de s'affranchir d'une modulation de fréquence parasite : la modulation est créée directement sur l'onde émise par la source et non sur la source elle-même. La variation d'impédance produite par la tension H.F. appliquée aux bornes d'une diode provoque une variation de la puissance d'hyperfréquence absorbée par la diode et par conséquent la modulation d'amplitude des micro-ondes.

Ce procédé de modulation présente les avantages suivants : faible puissance H.F. appliquée sur la diode (au maximum $20 \mathrm{~mW}$ ), donc faible rayonnement parasite, modulation bien sinusoïdale, taux de modulation suffisant (de l'ordre de $10 \%$ ) correspondant aux conditions théoriques, puissance modulée également suffisante (satisfaisant à la condition $a \ll 1$ ).

2.1.3. Référence de phase. - Le principe de la détection du signal $s$ est fondé sur la détection synchrone : il est donc nécessaire de posséder une référence de phase. Dans les trois bandes d'hyperfréquences considérées, cette référence est prise avant la tête de mesure sur le bras d'un coupleur à $20 \mathrm{~dB}$. Un atténuateur permet de faire varier la tension U.H.F. détectée, c'est-à-dire la tension de référence à la fréquence de modulation, pour la maintenir constante quelle que soit cette fréquence.

2.1.4. Tête de mesure. - La tête de mesure est l'organe où se produit l'excitation de la résonance de l'échantillon qui donne naissance au signal $s$. Dans le cas des bandes $X$ et $K a$, elle est constituée d'un guide rectangulaire terminé par un piston de court-circuit; la petite bobine captrice est placée à l'extérieur du guide, sur sa face large, et son couplage à l'échantillon situé dans le guide est rendu possible par un trou elliptique percé dans cette face (fig. 4 ). En bande $S$,

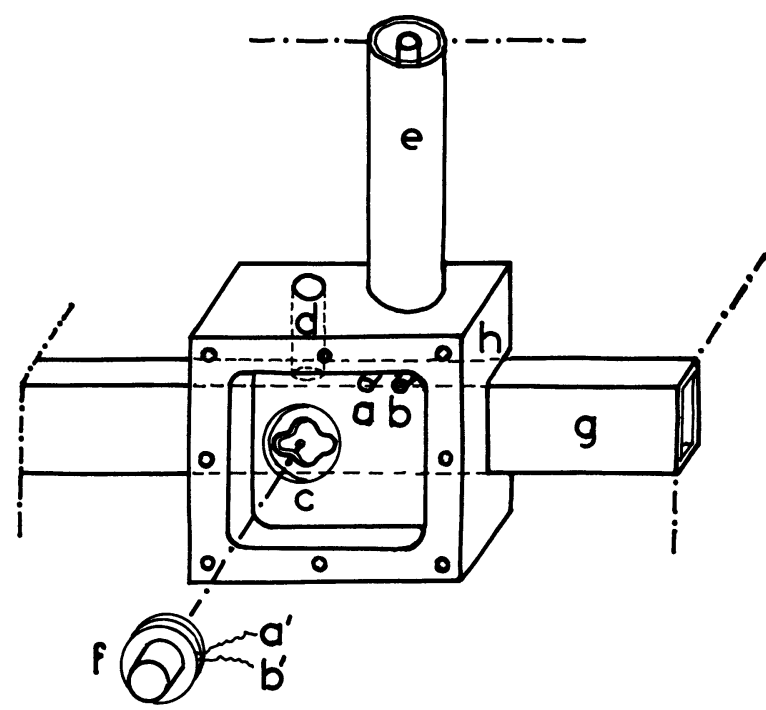

FIG. 4. - Vue en perspective, couvercle enlevé, de la tête de mesure en bande $K a$. La bobine de détection $\mathrm{f}$, dont les extrémités $\mathrm{a}^{\prime}$ et $\mathrm{b}^{\prime}$ sont soudées sur les bornes a et $b$ reliées au coaxial e, est placée sur l'orifice c percé dans le guide $8 \mathrm{~mm} \mathrm{~g}$. L'échantillon est introduit dans le trou d percé dans le blindage $\mathrm{h}$ et le guide $\mathrm{g}$. 
où la transmission des micro-ondes se fait par ligne coaxiale, l'échantillon se trouve placé dans une boucle et la petite bobine captrice a son axe dans le plan de la boucle et s'appuie sur elle. Dans les trois montages, un épais blindage protège la bobine de tout rayonnement extérieur.

2.2. Amplification et enregistrement dU Signal. - Le signal $s$ recueilli par la petite bobine est très faible : de l'ordre du $\mu \mathrm{V}$. Il faut donc l'amplifier et le transformer pour mesurer à la fois son amplitude et sa phase. Cette transformation se fait par un changement de fréquence qui permet de faire une double détection synchrone du signal en basse fréquence avec deux références, l'une en phase, l'autre en quadrature. En enregistrant ces deux signaux sur un enregistreur XY, on obtient exactement la représentation du vecteur de Fresnel du signal initial et, en faisant varier lentement le champ directeur, l'extrémité de ce vecteur décrit une courbe $S$.

2.2.1. Amplificateurs superhétérodynes. - Il s'agit d'abord d'amplifier séparément le signal et la référence. Chaque voie comprend un préamplificateur à large bande (la voie « signal » comporte en plus un étage à faible bruit), un changeur de fréquence à diodes à partir d'un oscillateur local commun et un amplificateur sélectif à double $\mathrm{T}$ accordé sur une fréquence intermédiaire de $30 \mathrm{kHz}$. La voie « référence " possède en plus un contrôle automatique de gain, pour maintenir constante la tension de référence appliquée aux détecteurs synchrones. L'ensemble de la chaîne d'amplification a un gain de $10^{6}$ pour la voie « signal » et de $10^{4}$ pour la voie « référence ", pour des fréquences d'entrée comprises entre $100 \mathrm{kHz}$ et $15 \mathrm{MHz}$. Nous avons cependant pu l'utiliser au-delà pour des fréquences allant jusqu'à $65 \mathrm{MHz}$. Sa bande passante est de $5 \mathrm{kHz}$ et son bruit propre ramené à l'entrée de $0,1 \mu \mathrm{V}$.

2.2.2. Détecteurs synchrones. - A partir du signal de référence amplifié à $30 \mathrm{kHz}$, que nous appellerons $R_{0}$, on élabore un second signal de référence $R_{\pi / 2}$ en quadrature avec le premier. Le signal de modulation est alors appliqué simultanément à deux détecteurs synchrones identiques ayant $R_{0}$ et $R_{\pi / 2}$ pour références. Les tensions continues fournies par ces deux détecteurs sont respectivement proportionnelles à $s \cos \psi$ et $s \sin \psi$, si on désigne par $s$ l'amplitude du signal $s(\Omega)$ et par $\psi$ sa phase par rapport à $R_{0}$. Elles sont alors appliquées aux entrées $\mathrm{X}$ et $\mathrm{Y}$ d'un enregistreur.

3. Caractéristiques pratiques de la méthode. Le problème qui se pose maintenant est de savoir comment, à partir de la courbe $\mathrm{S}$ d'un échantillon donné, tracée par l'enregistreur, on en déduit les valeurs de $T_{1}$ et de $T_{2}^{*}$ et avec quelle précision on les mesure. Il faut connaître également les valeurs limites des temps de relaxation mesurés et la sensibilité de la méthode. On aura alors décrit complètement cette nouvelle méthode de mesure des temps de relaxation.
3.1. Étalonnage. - La détermination du temps de relaxation $T_{2}^{*}$ est immédiate lorsque la courbe $S$ de l'échantillon est tracée : il suffit (cf. § 1.3) de mesurer l'angle $\Delta \varphi$. Mais la détermination de $T_{1}$, par la mesure de l'angle $\varphi$ (cf. $\S 1.4$ ), n'est possible que si l'on connaît la position de l'axe imaginaire. On l'obtient de deux façons différentes :

- En enregistrant à la même fréquence de modulation la courbe $S$ d'un corps de $T_{1}$ connu : on construit sa droite D et, puisque l'angle $\varphi$ est connu, on peut tracer l'axe imaginaire;

- En enregistrant à la même fréquence de modulation la courbe $S$ d'un corps de $T_{1}$ très court, beaucoup plus court que celui de l'échantillon à étudier : alors $\Omega T_{1} \ll 1$ et la droite $\mathrm{D}$ de ce corps étalon est confondue avec l'axe imaginaire.

En pratique, c'est ce dernier procédé que nous avons utilisé dans les expériences : la figure 5 donne un exemple de courbes $\mathrm{S}$ de divers échantillons en

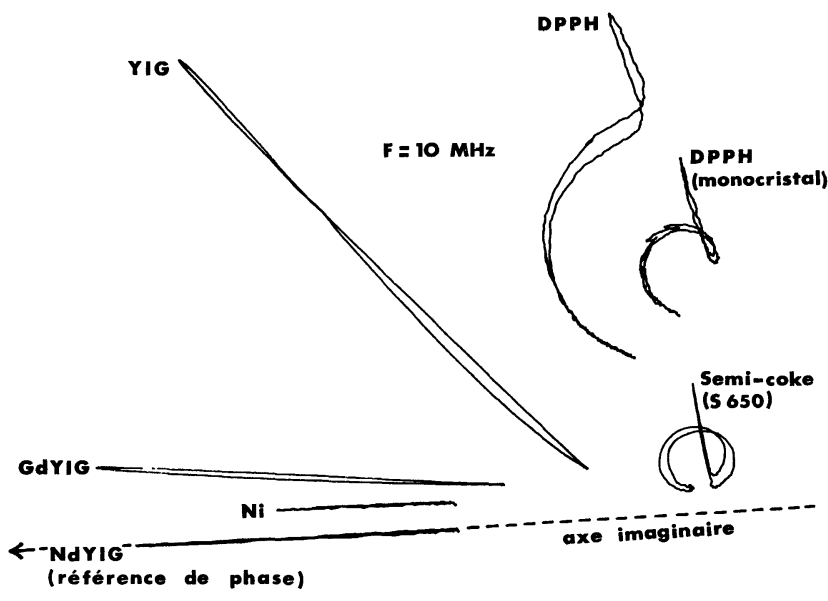

FIG. 5. - Exemples d'enregistrements de courbes S de différentes substances en poudre.

poudre et du choix de l'axe imaginaire. On remarque également sur cette figure la parfaite concordance entre les formes des courbes théoriques ( fig. 1 et 2 ) et expérimentales.

3.2. Précision. - Pour évaluer la précision de la méthode goniométrique, il faut tenir compte d'une erreur systématique due à l'approximation faite pour obtenir l'expression du signal $s$ etd'une erreur de mesure.

On montre que si les taux de modulation et de saturation ne sont plus infiniment petits, comme on l'a supposé précédemment, l'erreur systématique introduite est de l'ordre de $a / 2$, à condition que $k$ ne dépasse pas $20 \%$.

Quant à l'erreur de mesure, elle s'écrit, à partir des formules $(8)$ et $(10)$ et en appelant $\psi$ soit $\Delta \varphi$, soit $\varphi$, et $T$ soit $T_{2}$, soit $T_{1}$ :

$$
\frac{\mathrm{d} T}{T}=\frac{\mathrm{d}(\operatorname{tg} \psi)}{\operatorname{tg} \psi}+\frac{\mathrm{d} \Omega}{\Omega}=\frac{2 \mathrm{~d} \psi}{\sin 2 \psi}+\frac{\mathrm{d} \Omega}{\Omega} .
$$


L'erreur sur la fréquence est toujours négligeable $\left(<10^{-4}\right)$. Si l'on néglige l'erreur systématique, ce que l'on peut toujours faire en pratique, il reste celle sur les mesures d'angles que l'on peut évaluer à $\pm 1^{\circ}$, ce qui conduit pour $T$ à une erreur minimale, pour $\psi=45^{\circ}$ ou $\Omega T=1$, de :

$$
(\mathrm{d} T / T)_{\mathrm{m}}=(2 \pi / 180) \mathrm{d} \psi=3,5 \% \text {. }
$$

En revanche, si l'angle $\psi$ est très petit (ou, au contraire, très proche de $\left.90^{\circ}\right)$, l'erreur devient :

$$
(\mathrm{d} T / T)_{\mathrm{M}} \simeq \mathrm{d} \psi / \psi
$$

c'est-à-dire que, pour un angle de $2^{\circ}$, l'erreur faite sur $T$ est de $\pm 50 \%$. Si l'on veut mesurer les temps de relaxation avec une précision de $\pm 10 \%$, il faut choisir la fréquence de modulation telle que l'angle $\psi$ soit compris entre $10^{\circ}$ et $80^{\circ}$.

3.3. Gamme DES TEMPS DE RElaxation MESURÉs. Il est intéressant maintenant de connaître l'étendue de la gamme des temps de relaxation mesurés avec la précision indiquée précédemment. La fréquence de modulation peut, dans nos expériences, varier de $100 \mathrm{kHz}$ à $65 \mathrm{MHz}$. Avec une précision de $\pm 10 \%$, on a donc mesuré des temps de relaxation compris entre $9 \times 10^{-6}$ et $4,5 \times 10^{-10} \mathrm{~s}$. Mais la mesure peut s'étendre vers les temps de relaxation inférieurs à $10^{-10} \mathrm{~s}$, à condition d'augmenter la fréquence de modulation.

3.4. Sensibilité. - Il faut d'abord bien faire la différence qui existe entre la sensibilité d'un spectromètre classique, qui est la possibilité de détecter des raies pour mesurer par exemple leur facteur de Landé, et celle de notre montage, qui permet de mesurer des temps de relaxation avec une certaine précision. Dans notre cas, la sensibilité sera d'autant moins bonne que la précision exigée sera meilleure. Nous avons néanmoins fait le calcul de la sensibilité en conservant sa définition habituelle en résonance paramagnétique : elle s'exprime par le nombre de centres paramagnétiques qui donne un rapport signal sur bruit égal à l'unité. La précision n'intervient que dans l'application numérique.

En supposant que la bobine captrice est infiniment mince et a un rayon $r$ égal à sa distance à l'échantillon de volume $V$, on trouve que le nombre minimal de spins que le montage peut détecter avec un rapport signal sur bruit égal à l'unité est donné par :

$$
\begin{aligned}
& \left(N_{0} V\right)_{\min } \\
& \quad=\frac{32\left(\pi \mu_{0} \Delta F T_{1}\right)^{1 / 2}(r K T)^{3 / 2}}{a k(\gamma \hbar)^{2} H_{0} Q^{1 / 2}} \sqrt{\frac{\left(X^{2}+1\right)\left(Y^{2}+1\right)}{X\left(Y^{2}+4\right)}}
\end{aligned}
$$

où $\Delta F$ est la bande passante de l'appareil, $T$ la température et $Q$ le coefficient de surtension de la bobine supposée accordée.

La quantité sous le radical dépend des valeurs de $T_{1}$ et de $T_{2}$ :

- En supposant $T_{1} \gg T_{2}$ et avec $X=1$, on trouve :

$$
\left(N_{0} V\right)_{\min }=\frac{16\left(2 \pi \mu_{0} \Delta F T_{1}\right)^{1 / 2}(r K T)^{3 / 2}}{a k(\gamma \hbar)^{2} H_{0} Q^{1 / 2}}
$$

- En supposant $T_{1}=T_{2}$ et toujours avec $X=1$, on trouve alors :

$$
\left(N_{0} V\right)_{\min }=\frac{64\left(\pi \mu_{0} \Delta F T_{1}\right)^{1 / 2}(r K T)^{3 / 2}}{a k(\gamma \hbar)^{2} H_{0}(S Q)^{1 / 2}} .
$$

La différence entre ces deux résultats est minime. Nous avons fait le calcul dans le premier cas, en nous plaçant en bande $X$. En prenant les valeurs suivantes : $H_{0}=3300 \mathrm{G}, r=5 \mathrm{~mm}, Q=20, F=(1 / 2 \pi) \mathrm{Hz}$ (bande passante de l'enregistreur), $a=0,01$ et $k=0,1$, nous trouvons, en donnant à $T_{1}$ les valeurs limites mesurables par notre méthode (de $9 \times 10^{-6}$ à $\left.4,5 \times 10^{-10} \mathrm{~s}\right)$, que $\left(N_{0} V\right)_{\min }$ est compris entre $8 \times 10^{15}$ et $5 \times 10^{13}$ centres. En fait, pour que la mesure des temps de relaxation soit possible avec une précision de l'ordre de $\pm 10 \%$, il faut que le rapport signal sur bruit soit au moins égal à 10 . Dans ces conditions, le nombre de spins minimal utile varie entre $5 \times 10^{14}$ et $8 \times 10^{16}$, suivant les valeurs des temps de relaxation mesurés.

En conclusion, la méthode goniométrique est particulièrement intéressante pour les raisons suivantes :

- Elle permet de mesurer les temps de relaxation dans une gamme très étendue (de $10^{-5}$ à $\left.5 \times 10^{-10} \mathrm{~s}\right)$ avec une précision de $\pm 10 \%$.

- Elle est rapide, puisque deux enregistrements de " courbes de résonance » (du corps étalon et du corps à étudier) suffisent à déterminer $T_{1}$ et $T_{2}^{*}$. On peut encore la rendre plus rapide dans le cas où la mesure des temps de relaxation porte sur une famille de corps de temps de relaxation voisins : le même enregistrement comporte alors les courbes $S$ du corps étalon et de chaque corps de la famille, tracées successivement.

- Elle est surtout bien adaptée, par son principe même, à la mesure des temps de relaxation spinréseau très courts, ce qui jusqu'à présent n'était possible qu'avec une très mauvaise précision.

Remerciements. - Je tiens à exprimer mes plus vifs remerciements à MM. J. N. Aubrun et P. Veillet pour leur importante contribution à l'élaboration de cette méthode.

\section{BIBLIOGRAPHIE}

[1] Hervé (J.) et Pescia (J.), C. R. Acad. Sc., 1962, 255, 2926.

[2] Pescia (J.), Thèse, Paris, 2 juin 1964. 